\title{
Towards global architecture. The project between technique and technology
}

\author{
Alberto De Capua \\ Department of Architecture and Territory, Mediterranea University of Reggio Calabria, \\ e-mail: adecapua@unirc.it
}

\begin{abstract}
Environment, ecosystem, greenhouse effect emissions, public health, energy, sustainable development. This is an incomplete list of elements that are part of our daily lives, which show today's human footprint responsibility over the health of the planet. Although these issues have been for decades on all policy statements on development the situation is not improving. Indeed, constantly, we are called to change our lifestyle and our models of well-being that are causing an exaggerated and increasing waste of energy and resources, along with the increase of the overall impact of human species on natural systems. I do believe the environment is a resource that has been exploited and neglected for too long, however I am also convinced that excessive (ab)use of certain terms, such as the concept of sustainability, has weakened its driving force and innovation. There is no doubt that the thematic areas related to the monitoring of environmental quality and sustainable development dominate the cultural and socio-economic scenery for the articulation program design and governance of the territory, but the risk we are running today is to flatten themes of 'environment only for propaganda or even worse for the market. The report is a reasoning on innovation, technology and the complexity that now accompanies any work of transformation and that should lead to a "possible future".
\end{abstract}

Keywords: Sustainability, Technology, Project, Environment

\section{Introduction}

In today's overheated planet, where oil and gas reserves are not endless and where environmental emergencies/catastrophes can be found on our daily news, considering environmental issues should be the path to follow, but this seems to have lost its innovative impulse and is often subject to more economic reasons or category battles rather than a genuine will to improve the human condition. Especially if we see innovation as a technology transfer which is not aiming to solve today's problems, but follows a strategic perspective in relation to future scenarios.

In the construction industry, not immune to this global trend, the right tracks to innovation today are safety and sustainability. While on the first theme, recent dramatic news reports are an alarm bell that requires more attention and a new approach, where the share of responsibilities must be crystal clear, on the second theme, the right path to change the way we build and follow consistent approaches with sustainability models, is still unclear.

For several decades, themes related to sustainability have prevailed strongly in any discussion regarding the development models that our socially stigmatized society, on the brink of collapse, should follow. A debate that divides the good from the bad, judging individual behaviours, when speaking of people, and political strategies when speaking of nations or major business groups. 
Constant media warnings about the environmental impact, and the "ungrateful users" of the planet, are just as tiring and exhausting as the dramatic consequences we are inevitably going to face.

In fact, we cannot remain indifferent to what is happening, nor to the fact that these results were caused by incorrect models of development undertaken for more than half a century. It is truly shocking to see how signs of climate chaos are increasing, and far more obvious is the effect of extreme events which, together with uncontrolled human activities, increase the disasters our daily news is filled with.

Concerns for an immediate response are coming from all sides: as the economics Nobel prize Joseph Stiglitz drastically affirms "(...) if we do not find a way to limit environmental damage, to preserve energy and other natural resources, as well as to slow down the global warming, we are doomed to disaster (...), " and the illustrious Lester Brown that is already suggesting a "Plan B" for the environment, to reverse the trend; however, it seems to me that this represents at least two alarming facts:

- Plan "A" has already come to an end and probably hasn't produced the desired effects. We therefore expect, in a few decades, phases $\mathrm{C}, \mathrm{D}, \ldots$,

- There will be other "fashionable" recurrent terms yet to find in the near future, which international attention will surely be drawn to in the coming years, focusing on their meaning and most appropriate strategies to achieve their objectives.

In the past few years, there have been several suggestions on measures to take, unfortunately, too often unheard: Enzo Manzini wrote in 1997: "We are faced with a challenge: if we are to achieve sustainability, we need to increase the productivity of natural resources or dematerialize processes, products and services. "He hoped for a leapfrog - a quick step or a jump - to define the strategy to be pursued.

Or Hermann Daly, one of the founders of ecological economics, who suggested a recipe for a sustainable economy, also dismissed by the current economic model:

- Exploit renewable resources at a rate that does not exceed the regenerative capacity of the ecosystem.

- Limit the use of all resources, in order to produce a level of waste that can be absorbed by the ecosystem.

- Exploit the non-renewable resources at a pace that, as far as possible, does not exceed the pace of introduction of renewable substitutes.

However, these precautionary environmental strategies to increase eco-efficiency and reduce risks for humans and the environment, have never been so effective, and too often behind these actions is an economic growth intent rather than people's welfare.

\section{Sustainable society}

Contemporary culture conditioned by the greater technical and economic availability and greater independence from natural resources, has carried out transformation actions that a lot more destructive to the to the environment and do not meet urban quality standards nor the well-being of its inhabitants. The consequences of the changes have not produced, except for isolated cases, new ways of being, new development perspectives nor new fields for activities where one can apply creativity and social entrepreneurship.

Long-term strategies, that involve large investments and, despite their importance, have great difficulties to be implemented because they clash with the culture of the immediate that characterizes contemporary society, for this reason are often ignored.

After years of implementing the strategy for sustainable development it is now time to reflect on the progress made and how to carry out future strategies. 
Significant improvements have been made in all priority areas: incentives for energy efficiency buildings; measures for the greening of transport systems; improving the energy efficiency of buildings, revisions to the Directives on eco-design and ecolabelling, as well as progress has been made in research and funding. However, negative trends persist in several areas including the increasing demand for natural resources, the huge amount of waste the construction industry produces and the increasing consumption of energy in transport.

While, on the one hand it's clear that the international community's objective is to pursue sustainable development particularly focusing on global warming, on the other hand the market is shaping up around numerous investment initiatives by major financial and industrial corporations whose key is environmental performance of production processes and renewable energy sources. This is somewhat contradictory because we might find ecological battles on the same side of the barricade against the model of globalization together with the great empires who advocate production and financial sustainability concepts, by promoting choices and behaviours, despite the fact that there is no real control over the well-being of the planet and its people.

$\mathrm{CO}_{2}$ emissions have been increasing for years. At every summit, from Rio to Kyoto or Copenhagen, further ambitious goals are announced without any hope for concrete results. Indeed, we must have the courage to admit that we have actually made little progress so far. Edgar Morin, however, shows a wave of optimism: "If I were guided only by the light of reason, I should say that the world is moving towards the catastrophe that we are on the brink of the abyss. All the elements that we have under our eyes show us apocalyptic scenarios. But in the history of 'humanity there is the unexpected that changes the course of things. That's why, in the end, I am optimistic".

We develop some of our deepest social conflicts in the field of Globalization, and if properly managed, this phenomenon that can do a lot to improve the living conditions of both industrialized and developing countries. Although globalization critics are right to assert that it has been used as a tool to benefit certain values, nevertheless things can also change. Just as Stiglitz says, it's not written“ (...) in fact anywhere that globalization must be detrimental to the environment, increase social inequality, weaken cultural diversity and promote the interests of big corporations at the expense of the welfare of the common citizen. Today, the intellectual defense of the fundamentalism of the market has almost entirely disappeared.

According to Jeremy Rifkin the Third Industrial Revolution, which we are approaching, and the new era of "distributed capitalism" allow us to create a different approach to globalization, which this time focuses on the "continentalization from below", that allows us to acquire a "biosphere consciousness" and "global empathy" in time to prevent planetary collapse.

I do believe the environment is a resource that has been exploited and neglected for too long, however I am also convinced that excessive (ab)use of certain terms, such as the concept of sustainability, has weakened its driving force and innovation. There is no doubt that the thematic areas related to the monitoring of environmental quality and sustainable development dominate the cultural and socio-economic scenery for the articulation program design and governance of the territory, but the risk we are running today is to flatten themes of 'environment only for propaganda or even worse for the market. This is not meant to provoke nor to make a stand as those who always take the opposite side, yet it's a reasoning on innovation, technology and the complexity that now accompanies any work of transformation and that, without fear, should lead to a "possible future". 


\section{Sustainable building}

Our goal is to understand what is the complex system of principles that animates today's sustainable architecture. If only those addressed to a greater care and protection of the health of users or the environment and that also applies to social and economic issues at a time when change is proposed as a cultural, social, ecological and economic need for the protection of future generations. However, often, there is too little of this behind sustainable design, other than the adoption of eco-gadgets and market benefits. In fact, whilst the policy keeps following uncertain paths, the market is running fast: in the last five years, private investment in renewable and energy efficiency have been multiplied by ten. But there still is a big gap between the voluntary commitment of the market and that of the governments.

Our society is dominated by science and technology, however we only partly use these today to help us solve problems. It should be the obvious path to follow, rather than, as some may think, the practice by which the technique proceeds with greater freedom and becomes a form of careless handling of in-depth knowledge of the processes on which it operates.

In the building sector, increasing technical complexity of the product; the introduction of new technologies, too often poorly integrated with a structurally innovative production scenario; the lack of knowledge of building materials behaviours in relation to external actions and time; the hard measuring of the polluting effects of production also within our own buildings, should not lead us to a lack of confidence in the complexity produced by science and technology.

This should not be confused with the "technological optimism thesis", supported by traditional economists, according to which humanity will always be able to replace a resource shortage and increase the productivity of all kinds of energy and raw materials. I believe, in fact, unlike the supporters of this view, that complexity should not only be adapted to follow technological innovation in its most traditional means, but further explore suitability of clean technologies (eco-technologies and bio-compatible materials ).

Another misunderstanding is that the supporters of the existing "techno-centeredness" are accused of disenchant and little attention to the local context, often "drunk on unlimited growth."

Sustainable development must be the result of a synthesis between techno-scientific actions and socio-oriented policies based on a system of values.

Apart from slogans, the extremely difficult to manage existing complexity, cannot lead us into abandoning the idea that the new basic guidelines for architectural design, should take into consideration both the environmental impact and the effects on health and safety of its occupants. The need for greater scientific transparency is fundamental to avoid environmentalism dissolving into propaganda, rhetoric, or conformity. The environmental challenge issue is very complex because it involves different and complementary approaches and, above all, it must be handled by political instruments and international institutions. Therefore a more efficient technology and inter-disciplinary cooperation, are necessary conditions.

Today, technology, because of its nature and its trends, can play a central role in reducing the impact of production on the environment, although it remains the essence of its adaptation and transfer. As is the concern of high environmental impact of industrialization in developing countries, both in terms of environmental pollution, and depletion of nonrenewable natural resources.

Sooner or later, the world will have to consider introducing some changes before another series of global disasters. However changes, with no rules, that are implemented 
quickly in the wake of a crisis, aren't always the best way to reform the global economic system. The natural resource curse is not inevitable, but a matter of choice. The exploitation of natural resources, today, is an important part of globalization, and in some ways, as Stiglitz says, the failures of developing countries, that are rich in natural resources, represents the failures of globalization itself.

Thomas Friedman asserts that globalization and technology have flattened the world. Indeed, I believe, globalization has allowed, only in very rare cases, to reduce the gap between industrialized countries and so called emerging ones, hence not always enabling extraordinary opportunities for all. But this "flattening", which forces us to run faster and faster to keep up, has perhaps made our planet too small and too rapid to allow, each of us and society as a whole, to adapt permanently.

Architecture must focus on the inhabitant again, the citizen, in order to prevent seeing any proposed innovation as a solution to a specific problem, instead of a contribution to increasing the complexity of managing a space, a workplace, a city.

Initiating the right processes of technology to promote sustainable development means considering the technology not only as a set of tools, methods, techniques that allow us to implement changes in the physical world, bring it to an element of innovation and building value on being able to act human. Technology is also the engine to social innovation. Our goal should not be to control all the variables involved, but to reflect on the major indicators, on which sustainable quality achievement may depend on, within more traditional realization processes. Investigate new thematic areas, integrate existing ones with new specific requirements, combine the new indicators with the areas of application and the different levels of the project. Show designers the way to improve the environmental efficiency performance of the building and promote the use of adaptive technologies.

We should provide the designer with information about new needs to be met, leading the individual design choices towards awareness of natural balance, towards efficient transformation interventions, using technologies and tools to control the bioclimatic system. In these concepts, the term "efficiency" refers to the extent of the amount and frequency of exchanges at various levels of relationship between the physical and biological systems. Precise indicators are built-in the term; "Of contextuality", understood as the ability to recognize the place adjusting it and strengthening it, also in terms of resources supplied by the system; "Adaptability", meaning the ability of the system to adapt to environmental conditions (decide on technical and material options, technological innovation, experimentation, physical-technical contribution); "Duration", or ability to maintain/preserve in time; "Deconstruction", meaning the ability of the system to use, produce and recover resources.

\section{References}

1 Carson R., Silent Spring, Mariner Books, 1962.

2 Beck U. Risk Society: Towards a New Modernity, 1992, Sage Publication.

3 Bateson G., Mind and Nature: A Necessary Unity, Aug 2002, Hampton Press.

4 Davis M. Dead cities and other tales, The new Press, 2002.

5 Rifkin J. The Hydrogen Economy, J.P.Tarcher / Penguin, 2003.

6 Singer P. One World: The Ethics of Globalization, Yale University Press 2004.

7 Stiglitz J. Making Globalization Work, 2007 W.W. Norton \& Company. 
